\title{
THE CONTRIBUTION OF THE IERS TO ASTROPHYSICS AND GEODYNAMICS
}

\author{
M. Feissel \\ Central Bureau of IERS, Observatoire de Paris \\ 61, Avenue de l'Observatoire, 75014 Paris, France \\ Yaroslav Yatskiv, Main Astronomical Observatory, \\ Ukrainian Academy of Sciences, 2521267 Kiev 127, Ukraine
}

\begin{abstract}
The International Earth Rotation Service (IERS) maintains a celestial reference frame and a terrestrial reference frame based on observations in Very Long Baseline radio Interferometry, Lunar and Satellite Laser Ranging, and Global Positioning System, as well as a time series of the Earth Orientation Parameters in a system that is consistent at the level of $0.001 "$.

The IERS Celestial Reference Frame is based on positions of over 400 extragalactic objects, about 250 of which are known to better than \pm 0.0005 "; it will serve as absolute reference for galactic studies.

The IERS contributions to geodynamics cover crustal deformations as well as investigations of the dynamics of the solid and fluid layers of the Earth.

The topics covered in the talk are treated in a paper by M. Feissel and D. Gambis: The IERS: current results for research on Earth rotation and reference frames, Proc. of COSPAR meeting B.7-M (1992), "The orientation of the Earth as observed by modern techniques", to appear in Advances in Space Research (Pergamon Press).
\end{abstract}

